\title{
Effect of Temperature on the Synthesis of Nano-Nickel Particles
}

\author{
Haque KM A ${ }^{1, *}$, Huss ain M $S^{2}$, Alam S. $S^{1}$, Islam S. M. $S^{1}$ \\ ${ }^{1}$ Shahjalal University of Science and Technology, Sylhet-3114, Bangladesh \\ ${ }_{2}^{2}$ SIPCHEM, PO Box 130, Al-Khobar 31952, Kin gdom of Saudi Arabia
}

\begin{abstract}
The focus of this study is to observe the effect of temperature on the size and morphology of nano-nickel particles. Nano-n ickel particles have been prepared by a simple poly mer-surfactant interaction of a cationic poly mer poly vinyl pyrrolidone (PVP) with an anionic surfactant, sodium-dodecyl-sulphate SDS at different temperatures i.e. $60^{\circ} \mathrm{C}, 80^{\circ} \mathrm{C}$ and $100^{\circ} \mathrm{C}$. Nano-sized nickel particles were synthesized by using nickel chloride as the precursor, hydrazine as the reducing agent in the presence of SDS and PVP in a strong basic medium (pH-10.2). Our research shows that the rate of reduction increases as the reaction temperature was increased from $60^{\circ} \mathrm{C}$ to $100^{\circ} \mathrm{C}$. Finer particles of diameters less than $10 \mathrm{~nm}$ were formed as the temperature was increased from 60 to $100^{\circ} \mathrm{C}$. The morphology of the synthesized nano-nickel particles also varied as the temperature was increased. The concentration of nickel chloride and SDS/PVP was kept cons tant in all these experiments. The nano-sized nickel particles synthesized were characterized by using SEM and HRTEM.
\end{abstract}

Keywords Morphology, Hydrazine, Reduction, SEM, TEM

\section{Introduction}

In the past two decades, considerable attention has been devoted to the synthesis of metallic nano-particles specially nano-nickel particles because of their unusual properties that differ from either the bulk or single atoms. During the $19^{\text {th }}$ century and the first half of the $20^{\text {th }}$ century the preoccupation with metal colloids was essentially confined with in the academic communities as these materials offered excellent models for studying the relationships between the size, shapes and structure of the individual particles and their unique properties, such as , catalytic, optical, electrical and magnetic. During the second part of the last century, however, many of these properties have found applications in several fields of technology and as a result metallic particles ranging in size fro $m$ few nanometres to ten of micrometers are today used extensively in electronics [1-4], catalysis [5-8], pigments [9] and metallu rgy [10]. Several others applications such as bio-sensing[11-14], antimicrobial, non linear optics [15], high density magnetic storage[16], transparent conductive coatings[17] etc are still in early stages of development, but are expected to evolve rapidly within the next few years. More so than the traditional applications, these new technological fields require materials with controlled size, size distribution, morphology, composition, internal structure and surface characteristics. Consequently

* Corresponding author:

kazi_md_anam@yahoo.com (Haque K M A)

Published online at http://journal.sapub.org/nn

Copyright (C) 2012 Scientific \& Academic Publishing. All Rights Reserved there is a renewed interest in the development of preparation techniques capable of tailoring these properties needs to support the technical progress of the existing applications and meet the challenges of the emerging ones.

There are three different processes used for the synthesis of metallic nano-particles[18,19]. First process is "phase breakdown" in which by supplying energy necessary to increase the specific surface area of the dispersed matter, the size of the bulk metal can be reduced to the desired dimensions. This can be done by the mechanical breakdown of the solid metals using ball milling or by dividing the molten metal in to droplets, which are subsequently solidified by rapid cooling. These two methods are not capable of generating uniform or mono dispersed metallic particles. Second process is "phase transformation method" in which metal compounds are converted into finely divided metallic particles by thermal decomposition (thermolysis or pyrolysis) or chemical reduction. By this process it is possible to prepare uniform metal particles of different shape. Third process is "phase build-up", in this process metallic particles are constructed from their building blocks i.e. metal atoms. This may be in a gas phase i.e. chemical or physical vapour deposition or in a liquid phase i.e. chemical precipitation. Different techniques have been used to prepare the metal nano-particles, such as reduction of metal oxides and metal salts [20], sonochemical and thermal decomposition of metal comple xes[21] and reduction in solution by strong reducing agents [22].

We report here synthesis of nano-nickel particles by a phase transformation method in which $\mathrm{NiCl}_{2}$ is converted to nano sized Ni partic les through a chemical reduction process 
using hydrazine as a reducing agent along with a cationic polymer and an anionic surfactant in a basic medium. Surfactants-covered water pools offer a unique microenvironment for the formation of nano-particles. They do not only act as micro reactors for the processing reactions but also inhibit the excess aggregation of the particles because the surfactants could adsorb on the surfaces of the partic les when the particle size approaches that of water pool. As a result, the particles obtained in such a medium are generally very fine and monodispersed.

\section{Experimental}

Materials used for the synthesis of nano-nickel particles were nickel chloride analytical grade, sodium dodecyl sulphate, poly vinyl pyrrolidone, hydrazine hydrate solution sodium carbonate; de-ionized distilled water was used in the preparation of all the solutions. Instruments used for the preparation and characterization of nano-nickel particles were thermostatically controlled hot plate with magnetic stirrer, four necked round bottom flask, Condenser.

Nano-sized agglomerated Ni particles were synthesized by dissolving $8.78 \mathrm{~g}$ of $\mathrm{NiCl}_{2}(40 \mathrm{mmol})$ in a glass beaker containing $100 \mathrm{ml}$ of de-ionized distilled water at $40^{\circ} \mathrm{C}$. The $\mathrm{pH}$ of the solution was maintained at 10.2 by adding $\mathrm{Na}_{2} \mathrm{CO}_{3}$ solution to the reacting solution. At this point the temperature of the solution was raised to $60^{\circ} \mathrm{C}$ and $30 \mathrm{~mL}$ of hydrazine was added to the solution. In order to study the effect of temperature on the formation of nano-nickel particles, same experiment was carried out at $80^{\circ} \mathrm{C}$ and $100^{\circ} \mathrm{C}$.We observe that at $60^{\circ} \mathrm{C}$ as the reaction continues, the appearance of gray/black precipitates in the beaker meant that nickel particles have started to form. This reaction is not instantaneous and can take several hours for the nickel ion reduction to reach completion. The particles from bottom of the reaction vessel were collected, centrifuged (4000 rpm), washed with distilled water and ethanol for three times, and finally desiccated at room temperature before characterization. Scanning Electron Microscope (SEM) FEI-NOVA 200Nanolab with EDAX and High Resolution Transmission Electron Microscope (HRTEM) JEOL - JEM 2100F had been used to characterize these synthesized $n$ ickel partic les and elucidated results are discussed.

\section{Results and Discussion}

Metal ato ms are formed by transferring of electrons fro $\mathrm{ma}$ reducing agent $\left(\operatorname{Red}^{\mathrm{m}}\right)$ to the oxidized metallic species $\mathrm{M}^{\mathrm{n}+}$

$$
\mathrm{M}^{\mathrm{n}+}+\operatorname{Red}^{\mathrm{m}-\mathrm{M}^{0}+\mathrm{Ox}}{ }^{\mathrm{m}-\mathrm{n}}
$$

where $\mathrm{M}^{0}=$ metal atom produced by the gaining of electron from the reducing agent, $\mathrm{Ox}^{\mathrm{m}-\mathrm{n}}=$ species produced from the reducing agent after transferring electron to the metal ion, $\mathrm{m}$ and $\mathrm{n}$ are the charges. Transfer of electrons from the reducing agent to the metal ions mainly depends on the value of standard redox potential associated with the reaction $\Delta \mathrm{E}^{0}$.
For the information about mechanism of the reduction reaction, the value of $\Delta \mathrm{E}^{0}$ is also an excellent predictor of the rate of the chemical reaction. Generally an increase in its value is associated with a more rapid generation of atoms in the liqu id phase. $\mathrm{E}^{0}$ of equation (1) in a basic mediu $\mathrm{m}$ is -0.26 and for the equation (2) is -1.16 . So the value of $\Delta \mathrm{E}^{0}$ then becomes $-0.26-(-1.16)=+0.939 \mathrm{~V}$. The value of $\Delta \mathrm{E}^{0}$ for the reduction of by hydrazine in a basic mediu $\mathrm{m}$ is $+0.939 \mathrm{~V}$, so it is possible to reduce ion by hydrazine in the basic medium. $\mathrm{Ni}^{2+}$ ion can be reduced as follows:

$$
\mathrm{Ni}^{2+}+2 \mathrm{e} \rightarrow \mathrm{Ni}
$$

In a basic mediu mhydrazine acts as a very strong reducing agent

$$
\mathrm{N}_{2} \mathrm{H}_{4}+4 \mathrm{OH}^{-} \rightarrow \mathrm{N}_{2}+4 \mathrm{H}_{2} \mathrm{O}+4 \mathrm{e}
$$

So the overall reaction for the reduction of $\mathrm{Ni}^{2+}$ by hydrazine in a basic medium is as follows:

$$
\mathrm{Ni}^{2+}+\mathrm{N}_{2} \mathrm{H}_{4}+\mathrm{OH}^{-} \rightarrow \mathrm{Ni}+\mathrm{N}_{2}+\mathrm{H}_{2} \mathrm{O}
$$

In order to increase the $\mathrm{pH}$ to 10.2 concentrated sodium carbonate solution was added to the $\mathrm{NiCl}_{2}$ solution. However, as the sodium carbonate dissolves in water it forms $\mathrm{NaOH}$ which then react with $\mathrm{NiCl}_{2}$ and form $\mathrm{Ni}(\mathrm{OH})_{2}$

$$
\begin{gathered}
\mathrm{Na}_{2} \mathrm{CO}_{3}+\mathrm{H}_{2} \mathrm{O}=\mathrm{NaOH}+\mathrm{H}_{2} \mathrm{CO}_{3} \\
\mathrm{NaOH}+\mathrm{NiCl}_{2}=\mathrm{Ni}(\mathrm{OH})_{2}+\mathrm{NaCl}
\end{gathered}
$$

Hence, the $\mathrm{Ni}^{2+}$ ions from the compound $\mathrm{Ni}(\mathrm{OH})_{2}$ are reduced to Ni atoms as shown in equation (4).

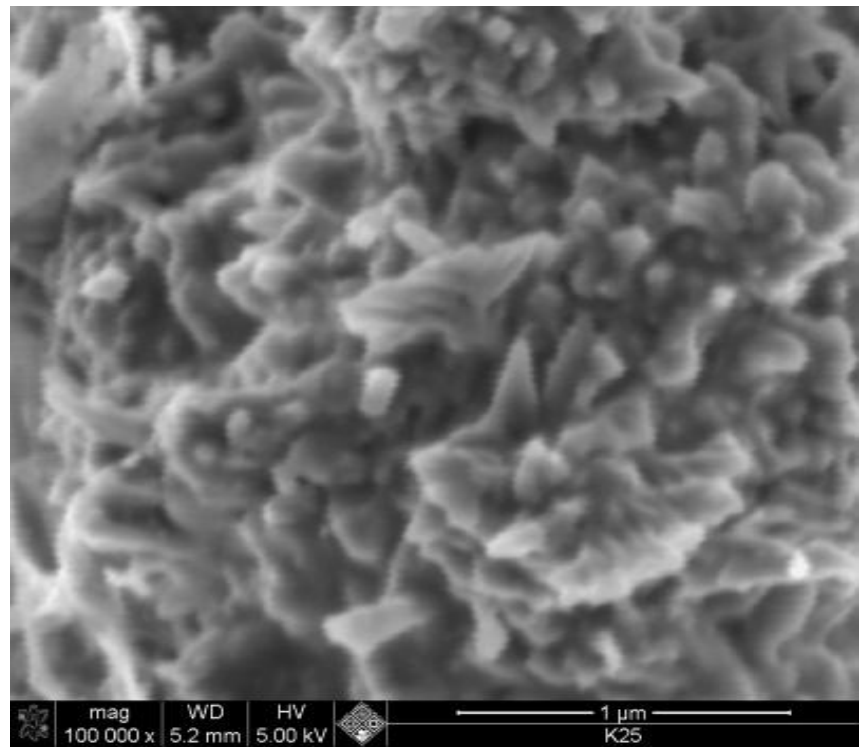

Figure 1. SEM image nano nickel particles synthesized at $60^{\circ} \mathrm{C}$, mag $\mathrm{x} 100,000$

Figure 1 shows SEM image of the nano-nickel particles that was synthesized using hydrazine with SDS and PVP at a reaction temperature of $60^{\circ} \mathrm{C}$. The SEM image shows the presence of spherical shaped particles.

Fig 2 shows SEM image of nano nickel particles synthesized using hydrazine with SDS and PVP at a reaction temperature of $80^{\circ} \mathrm{C}$. SEM image shows that the particles have both spherical and little spiky shaped.

Fig 3 shows SEM image of the nano-nickel particles which were synthesized using hydrazine with SDS and PVP at a reaction temperature of $100^{\circ} \mathrm{C}$. Here the SEM image 
shows that the particles have a kind of spiky morphology and there is a presence of hexagonal shaped structure. Experimental results show that by increasing the reaction temperature morphology of the synthesized nano-nickel particles changes from spherical, spherical spiky and to a combination of mixed hexagonal and spiky structures. As the temperature of the reaction is increased the kinetics of reaction is likely to increase with time. More newborn particles are nucleated by the reduction process and hence much finer particles can be expected to be formed.

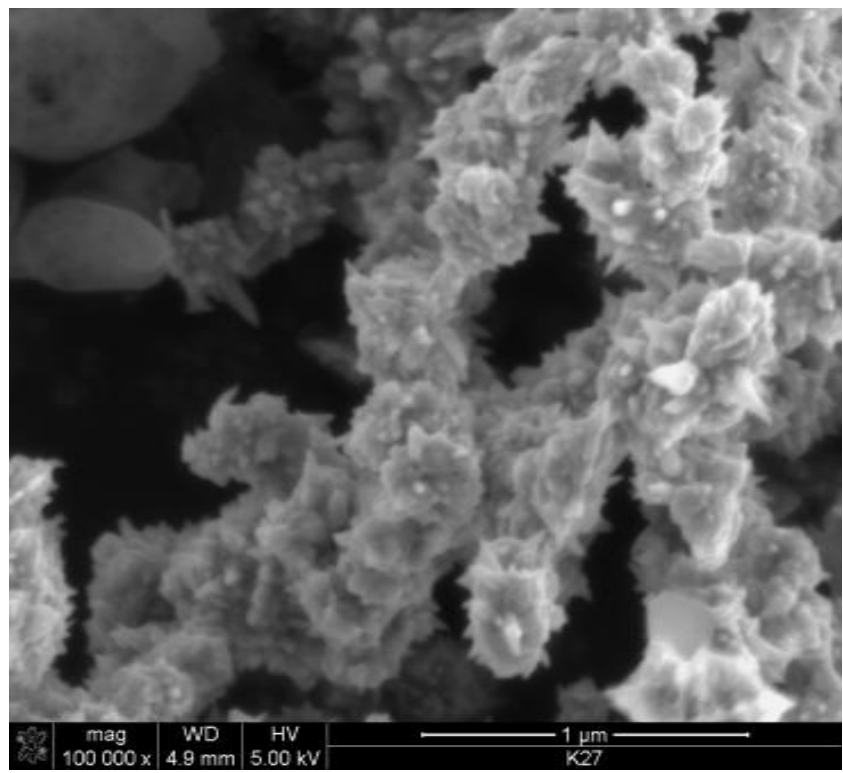

Figure 2. SEM image of nano nickel particles synthesized at $80^{\circ} \mathrm{C} \mathrm{mag} \mathrm{x}$ 80,000

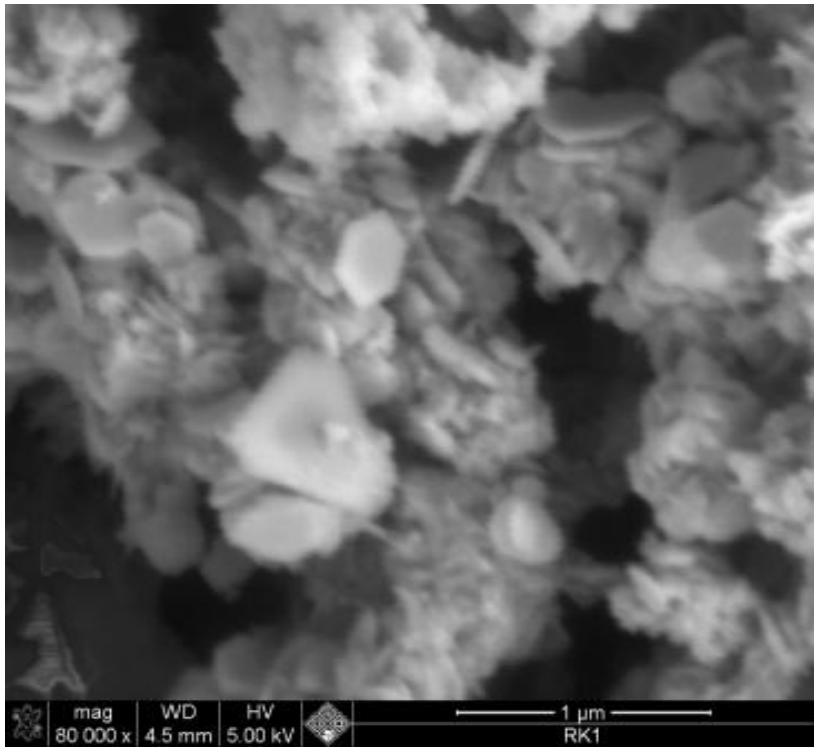

Figure 3. SEM image of nano nickel particles synthesized at $100^{\circ} \mathrm{C}$ mag $\mathrm{x} 80,000$

Figure 4 shows TEM image of the nano-nickel particles synthesized using hydrazine with SDS and PVP at a reaction temperature of $60^{\circ} \mathrm{C}$. Figure 4 shows that synthesized nano-nickel particles are around $31 \mathrm{~nm}$ in diameter. Size of the nickel nano-particles were determined using the scale bar at the bottom left of the figure 4 . Fig 5 shows image of the nano-nickel partic les synthesized using hydrazine with SDS and PVP at a reaction temperature of $80^{\circ} \mathrm{C}$. Figure 5 shows that synthesized nano-nickel particles are around $20 \mathrm{~nm}$ in diameter. Size of the nickel nano-particles are determined using the scale bar at the bottom left of the figure 5 .

Fig 6 shows TEM image of the nano-nickel particles that were synthesized using hydrazine with SDS and PVP at a reaction temperature of $100^{\circ} \mathrm{C}$. The image shows that synthesized nano-nickel particles are around $10 \mathrm{~nm}$ in diameter. Size of the nickel nano-particles are determined using the scale bar at the bottom left of the figure 6 .

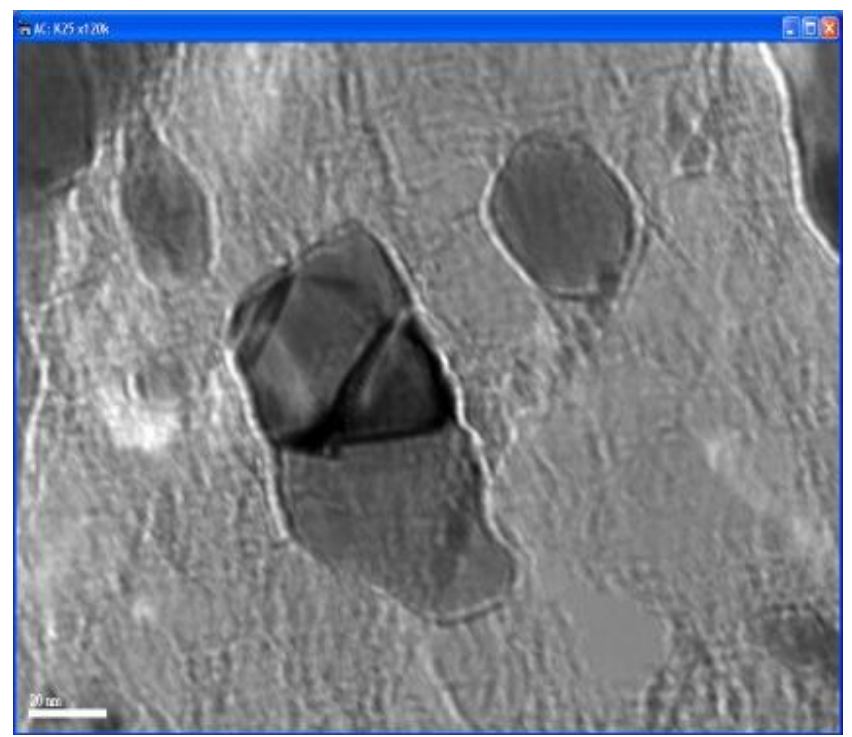

Figure 4. TEM image of nano-nickel particles synthesized at $60^{\circ} \mathrm{C}$

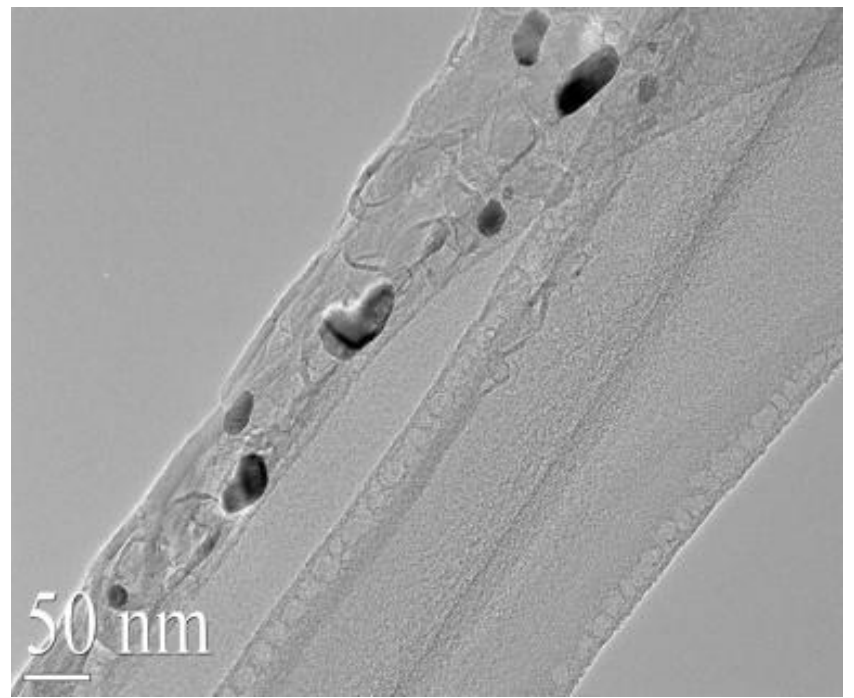

Figure 5. TEM image of nano-Ni particles synthesized at $80^{\circ} \mathrm{C}$

TEM images also show quite clearly that the diameter of the nickel particles had decreased considerably as the reaction temperature was increased from 60 to $100^{\circ} \mathrm{C}$. At increased temperatures not only the rate of nucleation would increase but one would also expect these Ni particles to have higher energy and hence move around with higher speed. Therefore we expect these particles would rather move 
around until they lose all the energy and as a consequence they would not get enough time to be agglomerated.

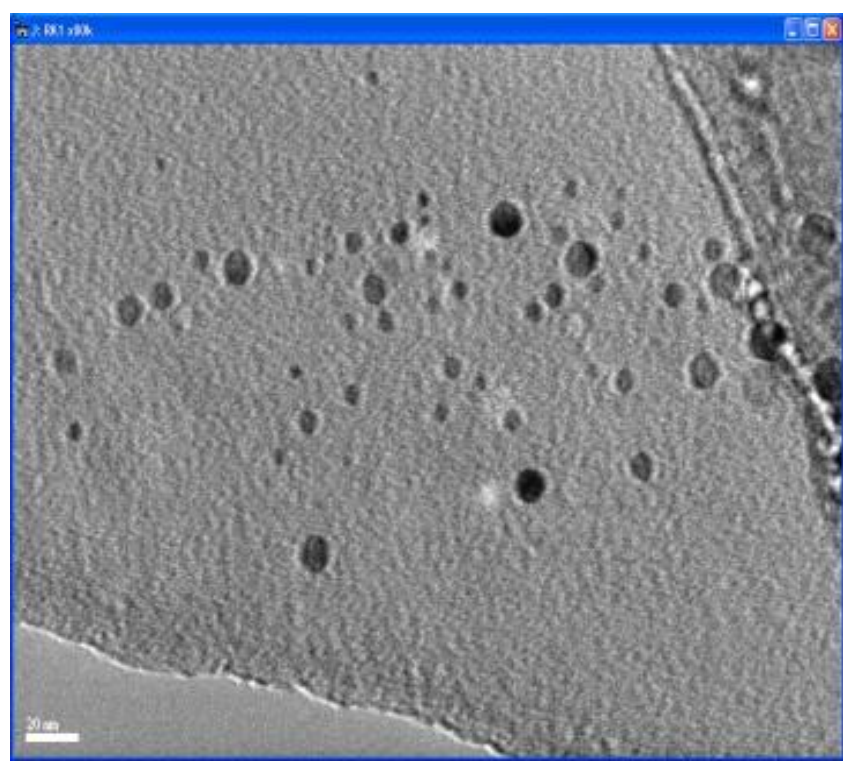

Figure 6. TEM image of nano-Ni particles synthesized at $100^{\circ} \mathrm{C}$

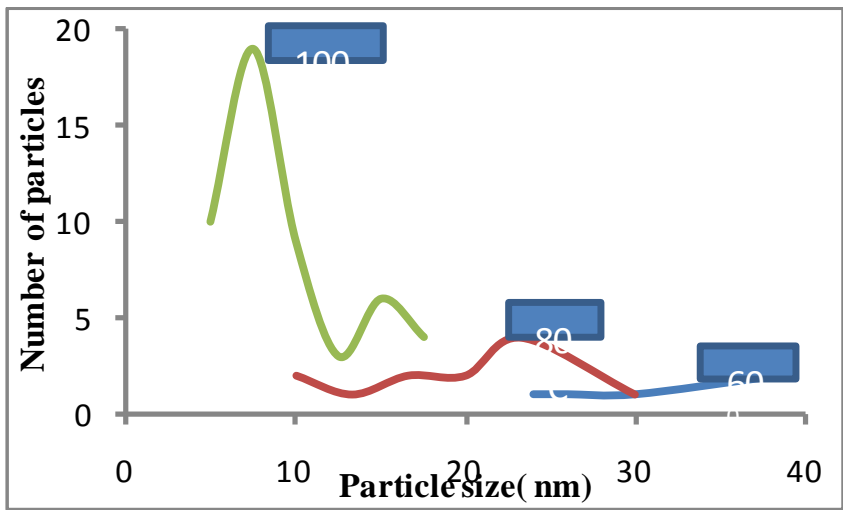

Figure 7. Plot of Particle size versus number of particles at different temperat ure

Figure 7 is the plot of particle size versus number of particles at different temperature. This plot shows the particle size distribution at different temperature and finest $\mathrm{Ni}$ particles were formed when SDS was used with PVP at a reaction temperature of $100^{\circ} \mathrm{C}$. The particle size formed were less than $10 \mathrm{~nm}$ in diameter (Fig 6). Ni particles synthesized at a reaction temperature of $80^{\circ} \mathrm{C}$ were around $20 \mathrm{~nm}$ in diameter (Fig 5). Ni particles synthesized at a reaction temperature of $60^{\circ} \mathrm{C}$ are around $31 \mathrm{~nm}$ in diameter (Fig 4). These results are summarized in Table 1.

Table 1. Effect of Temperat ure on the particle size

\begin{tabular}{|c|c|c|}
\hline Temperature & $\begin{array}{c}\text { Average } \\
\text { Particle size }\end{array}$ & Range \\
\hline $60^{\circ} \mathrm{C}$ & $31 \mathrm{~nm}$ & 14 \\
\hline $80^{\circ} \mathrm{C}$ & $19 \mathrm{~nm}$ & 23 \\
\hline $100^{\circ} \mathrm{C}$ & $10 \mathrm{~nm}$ & 13 \\
\hline
\end{tabular}

Nano-crystalline nickel has been synthesized by a chemical reduction process in which $\mathrm{NiCl}_{2}$ is converted to nano sized Ni partic les through a chemical reduction process using hydrazine as a reducing agent along with a cationic polymer and an anionic surfactant in a basic medium. Temperature has been found to play a significant role in the synthesis of nano-Ni particles. As the reaction temperature was increased from 60 to $100^{\circ} \mathrm{C}$, the particle size decreased. Smallest particles were formed at $100^{\circ} \mathrm{C}$. As the reaction continued these particles did not combine with neighbouring atoms and molecules to form clusters/agglomerates. It seems that the anionic surfactant had been able to provide the unique microenvironment required for the formation of nano-particles. This surfactant was able to minimize or inhibit the formation of excessive clusters/agglomerates of $\mathrm{Ni}$ atoms which were not able to form large scaled Ni particles/agglomerates. Using this chemical reduction process it has been possible to obtain nickel particles which were as fine as $10 \mathrm{~nm}$. Among currently available nano-particles, magnetic variants are used in a wide range of research area, including catalysis, biomedicine and magnetic resonance imaging (MRI), generally on the basis of their functional versatility. Nickel particles synthesized in this project has been used in the synthesis of NiCrAl alloy particles for aerospace applications.

\section{Conclusions}

Nano-sized nickel particles have been prepared by using a "phase transformation" method by using a chemical reduction process. In order to study the effect of temperature on the particle size, experiments were carried out at different temperatures i.e. 60,80 and $100^{\circ} \mathrm{C}$. SEM characterization showed that Ni particles synthesized at lower temperatures were almost spherical but as the temperature was increased particles showed very spiky morphology. However, finest $\mathrm{Ni}$ particles of less than $10 \mathrm{~nm}$ in diameter were formed at $100^{\circ} \mathrm{C}$. TEM characterization confirmed this inverse relationship i.e. Ni particle size decreased as the reaction temperatures were increased from 60 to $100^{\circ} \mathrm{C}$. The focus of this study is in the applications of nano-Ni particles in aerospace and med ical/biological applications.

\section{ACKNOWLEDGMENTS}

The authors would like to thank the financial support received from King Abdul Aziz City for Science and Technology (KACST), Riyadh, Saudi Arabia under Project No. 26-05.

\section{REFERENCES}

[1] D. W. Hamer and J. V. Biggers, Thicks Film Hybrid Microcircuit Technology, Wiley-Interscience, New York, 1972.

[2] J. G. Pepin, J Mater. Sci, 1991, 2, 34- 39 
[3] D. V. Goia, I Burn and G.Varga, Advanced Technologies of Particles synthesis for Electronics applications. ECCE-European Congress of Chemical Engineering, June26-28, 2001, Nuremberg, Germany.

[4] M. D. Musik, D. C. Keating, M. H. Keefe and M. J. Natan. Chem. Mater, 1997, 9, 1499-1507.

[5] J. P. Wilcoxon, A. Martino, R. L. Baughmann, E. Klavetter and A. Sylwester, Mater. Res. Soc. Symp. Proc., 1993, 286, 131.

[6] H. Bonnemann and W. Brijoux, in Advanced Catalysis and Nanostructered Materials, ed. W. R. Moser, Academic Press, San Diago, 1996, p 165.

[7] G. W. Busser, J. G. Van Ommen and J. A. Lercher in Advanced Catalysis and Nanostructered Materials, ed. W. R. Moser, Academic Press, San Diago, 1996, p213.

[8] J. F. Hamilton and R. C. Baetzold, Science, 1979, 205, $1213-$ 1220

[9] R. Vogt, K. Bernh ard and G. Plaff, (Merck, Germany), US pat. 5702518, Dec. 30, 1997.

[10] P. B. Joshi, N. S. S. Murti and V. L. Gadgeel, J Mater Sci. Lett., 1995, 14, 1099-1101.

[11] C. A. Markin, R. L. Letsinger, R. C. Mucic and J. J. Storhoff, Naty re, 1996, 382, 607-609.
[12] L. A. Lyon, M. D. Musick and M. J. Natan, Anal. Chem., 1998, 70, 5177- 5181 .

[13] M. J. Nathan and L. A. Lyon, in Metal Nano particles : Sy nthesis, Characterization and Applications, ed D. L. Feldheim and C. A. Foss, Marcel Dekker Inc., New York, 2002, p183.

[14] R. Elghanian, J. J. Storhoff, R. C. Mucic, R. L. Letsinger and C. A. Mirkin, Science, 1997, 277, 1078.

[15] R. C. Jhonson and J. T. Hupp, in Metal Nano particles : Synthesis, Characterization and Applications, ed D. L. Feldheim and C. A. Foss, Marcel Dekker Inc., New York, 2002, $\mathrm{p} 141$.

[16] S. Sun and C. B. Murray, J Appl. Phy s. 1999, 85, 4325-4330.

[17] N. Takamiya, Y. Kunimitsu, K. Adachi, K Mori,I. Noda,and A. Wakabay ashi, US pat 6143418, Nov7, 2001.

[18] D. V. Goia J Materials Chemistry, 2004, 14, 451-458.

[19] M S Hussain, Nanodeposition Research Project, KACST, Project No. 26-05, Final Technical Report, September 2007.

[20] S. C. Davis and K. J. Klabunde, Chem. Rev. 1982, 82, 157.

[21] D. E. Zhang, X. M. Ni, H. G. Zheng, Y. Li, X. J.Zhang, and J. P. Yang. Materials Letters 2005, 59, 2011-2114.

[22] C. Petit, P. Lixon, M, P. Pileni, J. Phys. Chem.1993, 97, 12974. 